\title{
The translational neural circuitry of anxiety
}

\author{
Oliver J Robinson 다, 1,2 Alexandra C Pike 지, 1,2 Brian Cornwell, ${ }^{3}$ Christian Grillon ${ }^{4}$
}

- Additional material is published online only. To view please visit the journal online (http://dx.doi.org/10. 1136jnnp-2019-321400).

${ }^{1}$ Institute of Cognitive Neuroscience, University College London, London, UK

${ }^{2}$ Research Department of Clinical, Educational and Health Psychology, University College London, London, UK

${ }^{3}$ Swinburne University of Technology, Hawthorn, Victoria, Australia

${ }^{4}$ National Institute of Mental Health, Bethesda, Maryland, USA

\section{Correspondence to} Dr Oliver J Robinson, Institute of Cognitive Neuroscience, University College London, London WC1N 3AR, UK; 0. robinson@ucl.ac.uk

Received 12 June 2019

Revised 17 June 2019

Accepted 17 June 2019

Published Online First 29 June 2019

Check for updates

(C) Author(s) (or their employer(s)) 2019. No commercial re-use. See rights and permissions. Published by BMJ.

To cite: Robinson OJ, Pike AC, Cornwell B, et al. J Neurol Neurosurg Psychiatry 2019:90:1353-1360.

\section{ABSTRACT}

Anxiety is an adaptive response that promotes harm avoidance, but at the same time excessive anxiety constitutes the most common psychiatric complaint. Moreover, current treatments for anxiety-both psychological and pharmacological-hover at around $50 \%$ recovery rates. Improving treatment outcomes is nevertheless difficult, in part because contemporary interventions were developed without an understanding of the underlying neurobiological mechanisms that they modulate. Recent advances in experimental models of anxiety in humans, such as threat of unpredictable shock have, however, enabled us to start translating the wealth of mechanistic animal work on defensive behaviour into humans. In this article, we discuss the distinction between fear and anxiety, before reviewing translational research on the neural circuitry of anxiety in animal models and how it relates to human neuroimaging studies across both healthy and clinical populations. We highlight the roles of subcortical regions (and their subunits) such as the bed nucleus of the stria terminalis, the amgydala, and the hippocampus, as well as their connectivity to cortical regions such as dorsal medial and lateral prefrontal/cingulate cortex and insula in maintaining anxiety responding. We discuss how this circuitry might be modulated by current treatments before finally highlighting areas for future research that might ultimately improve treatment outcomes for this common and debilitating transdiagnostic symptom.

\section{INTRODUCTION}

Fear and anxiety are adaptive, defensive reactions to threat across species. However, excessive fear or anxiety can interfere with quality of life. Indeed, anxiety disorders are the most prevalent psychiatric disorders, and excessive anxiety is implicated in most psychiatric disorders, as well as a number of other medical and neurological conditions. Anxiety is thus accompanied by a high financial cost. ${ }^{1}$ Compounding this, response rates to first-line pharmacological and psychological treatments are less than $50 \%{ }^{2}$ : most patients fail to respond to the first treatment that targets their anxiety. Other review papers focus on the neural circuitry of fear $^{3-5}$; this review focuses on our understanding of the underlying neurobiology of anxiety, as a construct not only more closely related to anxiety disorders such as generalised anxiety disorder (GAD) but also highly relevant transdiagnostically to other psychiatric and neurological disorders. In this review, which is intended as a broad narrative introduction for those new to the field, we argue that understanding this neurobiology, as well as the features that differentiate adaptive from pathological anxiety, is key to identifying pathological mechanisms and treatment targets.

\section{FEAR AND ANXIETY}

While fear and anxiety share many subjective and physiological symptoms, they can be differentiated based on behavioural profiles determined by certainty, ${ }^{67}$ which can be further subdivided into the contingency, temporal precision and spatial precision of the threat:

- In fear, the danger is imminent, unambiguous and mobilises the organism to take immediate action. Fear is above all a rapid behavioural response that leads to active avoidance (e.g. fight-or-flight) or other automatic responses, such as freezing in prey animals or piloerection (goosebumps). Pathological fear is seen in specific phobias, which are characterised by a marked fear of specific objects.

- In anxiety, the threat is more diffuse and uncertain. Anxiety is a lasting state of apprehension of potential future threats, accompanied by negative affect, autonomic symptoms, worry, increased vigilance and passive avoidance. ${ }^{8}$ Excessive anxiety symptoms can be found in GAD and panic disorder (PD) (though panic attacks themselves may be better characterised by models of acute fear, PD (including apprehension of subsequent attacks) is considered to be better modelled by anxiety). ${ }^{8}$

There is a body of research indicating that fear and anxiety are tractable and are associated with distinct pathologies. ${ }^{679}$ Early research into fear and anxiety found that there was a double dissociation between neural structures relating to threats that were phasic (fear) and sustained (anxiety), ${ }^{6}$ which led to a theoretical model in which anxiety and fear are putatively separate processes. Although the true neurobiological picture is likely to be more nuanced than this, ${ }^{10} 11$ this distinction is also reflected in the Research Domain Criteria (RDoC) matrix, which distinguishes between acute threat (fear) and potential threat (anxiety) ${ }^{12} 13$ as well as in the Diagnostic and Statistical Manual of Mental Disorders, Fifth Edition (DSM-5) where specific phobias are defined as 'cued by the presence or anticipation of specific objects or situations', while GAD, by contrast, is defined as 'Excessive anxiety and worry (apprehensive expectation) ... about a number of events or activities' ${ }^{14}$

The widest array of research to date involves Pavlovian cued fear conditioning in rodents. ${ }^{15}$ Unfortunately, while fear conditioning is a useful model of fear, it is insensitive to drugs that are anxiolytic in humans ${ }^{16-19}$ and is thus a poor model of anxiety disorders such as GAD. ${ }^{20} 21$ Comparatively 
little is known about anxiety, especially its many human-specific cognitive-affective features. Indeed, many animal models of anxiety, such as the elevated-plus maze, have few analogues in humans $^{22}$ (although see Biederman et $a l^{23}$ and Bach et $a l^{24}$ ), and of course the impact of psychological therapies cannot be studied in animals. However, innovative approaches to study anxiety experimentally in humans have recently been developed. This article reviews this emerging literature and suggests a model of its neural underpinnings.

\section{PHENOMENOLOGY OF PATHOLOGICAL ANXIETY}

Following Freud, who distinguished chronic anxiety from anxiety (panic) attack, clinicians have long recognised that anxiety is not a unitary phenomenon. ${ }^{8}$ This non-unitary view of anxiety is reflected in the DSM-5, which identifies several anxiety disorders characterised by shared 'features of excessive fear and anxiety', including PD, GAD, social anxiety disorder and simple phobia. Other disorders within the DSM also have anxiety as a core symptom, such as obsessive-compulsive disorders and addiction disorders. Additionally, a number of neurological disorders feature elevated anxiety, including variants of dementia such as frontotemporal dementia, vascular dementia and Alzheimer's disease, ${ }^{25}$ along with Parkinson's disease ${ }^{26}$ and traumatic brain injury. ${ }^{27} 28$

However, despite symptom heterogeneity, we do not at present have clear objective markers that can differentiate between disorders which feature anxiety, and there is, moreover, strong symptom overlap. All share common enduring behavioural, cognitive and physiological characteristics, potentially arising from impairments in transdiagnostic features, as seen in the $\mathrm{RDoC}^{12}$ domain of negative valence systems ${ }^{13}$ which highlights exaggerated or problematic responses to 'potential threat (anxiety)'. In this article, based on the assumption that similar phenomenological presentations of 'anxiety' reflect true underlying neurobiological similarities, we discuss the shared neural circuitry which may underlie sustained anxiety symptoms.

\section{TRANSLATIONAL NEUROSCIENCE OF ANXIETY}

Early work in fear conditioning in animal models highlighted the key roles of two amygdala nuclei, the basolateral amygdala (BLA) and the central nucleus of the amygdala (CeA), in anxiety. The BLA integrates sensory information from the environment and, via its projections, excites the CeA. The amygdala subsequently triggers defensive responses via efferent projections to regions such as the stria terminalis, the hippocampus, the ventral striatum, the orbitofrontal cortex, the periaqueductal gray and the hypothalamus. ${ }^{29}$ While the amygdala is important for fear conditioning, its direct role in maintaining sustained anxiety symptoms has been more difficult to establish. Lesions of the amygdala do not reduce defensive responses in models of anxiety such as the elevated plus-maze ${ }^{30}$ and the anxiolytic benzodiazepine does not act via the amygdala. ${ }^{31}$

This contrasts with another structure tightly coupled with the CeA, the bed nucleus of the stria terminalis (BNST), which does appear to be preferentially involved in maintaining sustained anxiety. ${ }^{6732}$ The BNST is a part of the 'extended amygdala', 33 which is well-situated to regulate defensive responses such as anxiety via its GABA (gamma-aminobutyric acid)-ergic projections to various limbic, hindbrain and cortical structures. ${ }^{6732}$

Consideration of the conditions that determine the involvement of the BNST in defensive responses emphasises the role of temporal unpredictability of the threats ${ }^{34}$ and the sustained duration of the response. ${ }^{75}$ Early evidence of a differentiation
Table 1 Studies informing our emerging understanding of the functions of the subregions of the BNST

\begin{tabular}{|c|c|c|}
\hline Region of BNST & Function & Species \\
\hline Right anterior & $\begin{array}{l}\text { Activated during threat }>\text { safe contrast in healthy } \\
\text { control participants }{ }^{40}\end{array}$ & Human \\
\hline Anteroventral & $\begin{array}{l}\text { Paraventricular nucleus excitation, promotes } \\
\text { corticosterone secretion }^{41}\end{array}$ & Rat \\
\hline Posterior & $\begin{array}{l}\text { Inhibits stress-induced paraventricular nucleus } \\
\text { excitation, adrenocorticotropic hormone release and } \\
\text { plasma corticosterone responses }{ }^{41}\end{array}$ & Rat \\
\hline $\begin{array}{l}\text { Anterolateral } \\
\text { (encompassing } \\
\text { oval nucleus) }\end{array}$ & $\begin{array}{l}\text { Attenuates stress-induced reductions in weight gain at } \\
\text { the end of } 7 \text {-day stress period: mediating consequences } \\
\text { of repeated stress? }\end{array}$ & Rat \\
\hline Anterodorsal & $\begin{array}{l}\text { Social behaviour, namely defensive and reproductive } \\
\text { behaviour }^{43} ; \text {; promotes anxiolytic behaviour, for } \\
\text { example, decreased risk avoidance, decreased } \\
\text { respiratory rate, positive conditioning valence }{ }^{38}\end{array}$ & Rat \\
\hline Oval nucleus & Anxiogenic (perhaps by inhibiting anterodorsal BNST) ${ }^{38}$ & Rat \\
\hline Ventral & $\begin{array}{l}\text { Innervates VTA, stimulating glutamatergic projections } \\
\text { results in aversive/anxiogenic phenotypes, stimulating } \\
\text { GABAergic projections results in rewarding/anxiolytic } \\
\text { phenotypes by the inhibition of VTA GABAergic } \\
\text { neurons }{ }^{39} \text {; noradrenergic neurons may control freezing } \\
\text { behaviour }{ }^{44}\end{array}$ & Rat \\
\hline
\end{tabular}

Note that the majority of work has been done in rodents, due to the lack of spatial resolution of MRI prior to the advent of 7T MRI scanning.

BNST, bed nucleus of the stria terminalis; GABA, gamma-aminobutyric acid; VTA, ventral tegmental area.

between fear and anxiety in the BNST came from studies using the startle reflex. 'Fear-potentiated startle' refers to the increased startle reflex amplitude in the presence of a short-duration threat, whereas 'anxiety-potentiated startle' refers to the increased startle amplitude during a long-duration unpredictable threat. A series of studies by Davis and collaborators established a double dissociation between the CeA and BNST; lesions of the CeA abolish fear-potentiated startle, but not anxiety-potentiated startle, while lesions of the BNST suppress anxiety-potentiated startle, but not fear-potentiated startle. ${ }^{67}$ According to Davis' group, anxiety is thus maintained by activation of corticotrophin receptors in the BNST. $^{7}$

More recent studies have, however, provided evidence that there is more nuance to the role of the BNST in anxiety. This is perhaps unsurprising given that the BNST is small but heterogeneous, with up to 18 functionally distinct subregions. ${ }^{36}$ Some BNST lesions can $u p$-regulate anxiety, ${ }^{37}$ while optogenetic stimulation of discrete BNST subregions can down-regulate anxiety. ${ }^{38} 39$ Different efferent connections of the BNST also control different features of anxiety, ${ }^{38} 39$ suggesting that distinct BNST subregions dynamically control different aspects of defensive behaviour ${ }^{38-44}$ (see table 1). Of note, the story is almost certainly the same with regards to the role of the amygdala in fear, which is gradually being broken down into component microcircuits. 4546

Moving into the cortex, the medial prefrontal cortex (mPFC) is implicated in attention and affective information processing ${ }^{47}$ and hence also involved in responses to threat. The rodent mPFC is composed of several subregions including the infralimbic (IL) and prelimbic (PL) cortex, which are densely interconnected with the amygdala ${ }^{48}$ and play opposing roles in defensive behaviours, ${ }^{49}$ with the IL inhibiting the amygdala (and defensive behaviours) and the PL exciting the amygdala. Empirical evidence of a role for the PL in sustained anxiety has been established using a wide range of paradigms including the elevated plus-maze and the open field test, ${ }^{50}$ context conditioning ${ }^{51}$ and 
fear conditioning with long-duration conditioned stimuli (CS). ${ }^{52}$ Electrophysiological recordings in rodents show that PL neurons maintain persistent firing that correlate with freezing throughout the duration of sustained threat ${ }^{5152}$ and that PL firing persists during trace fear conditioning, ${ }^{53}$ a paradigm during which the unconditioned stimulus (US) is delivered after an empty interval following the end of the CS. These results suggest PL involvement in both defensive behaviours and the neural representation of threat (ie, US).

The hippocampus also conveys contextual information about environmental threat ${ }^{54}$ to the PL. ${ }^{55}$ Specifically, brain structures communicate via synchronised activity both in local networks ${ }^{56}$ and over longer distances. ${ }^{57}$ This can be observed in theta oscillations, which have been linked to anxiety in rodents and humans ${ }^{58}$ and display synchronised activity between the ventral hippocampus and PL in anxiogenic contexts. ${ }^{54}$ Additionally, research using operant conflict tasks has suggested that the hippocampus has a key role in decision-making in situations where there is a conflict between approaching rewards and avoiding punishments, a scenario which induces anxiety across species. ${ }^{59}$

Interoceptive information from visceral changes may also be conveyed, via the anterior insula, to the PL. ${ }^{60}$ Specifically, robust visceral changes (heart rate, gastrointestinal, blood pressure) caused by anxiety may generate a feedback loop between the PL and anterior insula that contributes to the maintenance of anxiety. Threat representation in the PL could then influence anxiety responses via direct ${ }^{\mathrm{s} 1}$ or indirect input to the BNST through the BLA. ${ }^{60}$

Finally, prefrontal cortex (PFC) regions are also involved in anxiety via their role in working memory (WM). In rodents, WM deficits are associated with increased anxiety. ${ }^{\text {s2 }}$ Primates have larger prefrontal cortices with additional medial and lateral dissociations than rodents. In monkeys, as in humans, WM relies in part on the dorsolateral PFC (dlPFC), ${ }^{\mathrm{s} 3}$ a structure that is also implicated in anxiety and stress. Maternal separation stress in young monkeys activates the right dlPFc, but deactivates the left dlPFC. ${ }^{4}$ In young monkeys, as in children, heightened reaction to novelty and potential threat characterises an anxious temperament disposition, which is a well-established risk for the development of anxiety. ${ }^{\mathrm{s5}}$ Critically, such anxious disposition in monkeys is associated with dIPFC malfunction. ${ }^{\text {s6 }}$

A possible neural model of anxiety that emerges from these studies in animals is that environmental signals from the ventral hippocampus and interoceptive signals from the anterior insula help maintain threat representation in the PL, which is then used to guide defensive behaviours via the BNST and the amygdala and may be under the control of the dlPFC (see figure 1).

\section{NEUROSCIENCE OF HUMAN ANXIETY AND ITS PATHOLOGY}

In recent years, innovative translational experimental models that attempt to mimic and quantify sustained anxiety in humans have emerged. These include darkness ${ }^{\mathrm{s} 7}$, low concentration $(7.5 \%)$ of $\mathrm{CO}_{2}$ inhalation $^{\mathrm{s} 8}$ and vigilant threat monitoring ${ }^{\mathrm{s} 9}$ (see table 2). However, one widely used anxiety model in humans relies on long-duration threat of aversive stimuli such as shock (i.e. threat of shock or context conditioning ${ }^{\text {s10 }}$ ), where subjects are informed that shocks may be delivered unpredictably.

Other paradigms investigating fear (eg, cued fear conditioning ${ }^{\text {s11 }}$ ) may seem at first sight to be investigating similar constructs. However, these paradigms are generally much more precise as to the temporal (and spatial) precision of the shock association (notably, further research is needed to ascertain the precise temporal/spatial conditions under which anxietyrelated-rather than fear-related-circuitry is activated).

One key advantage of the startle probe methodology, discussed in the animal section above, is that it can also be employed in humans. Healthy human participants display heightened startle sensitivity during unpredictable threat relative to baseline, but, importantly, this startle sensitivity is further elevated in those

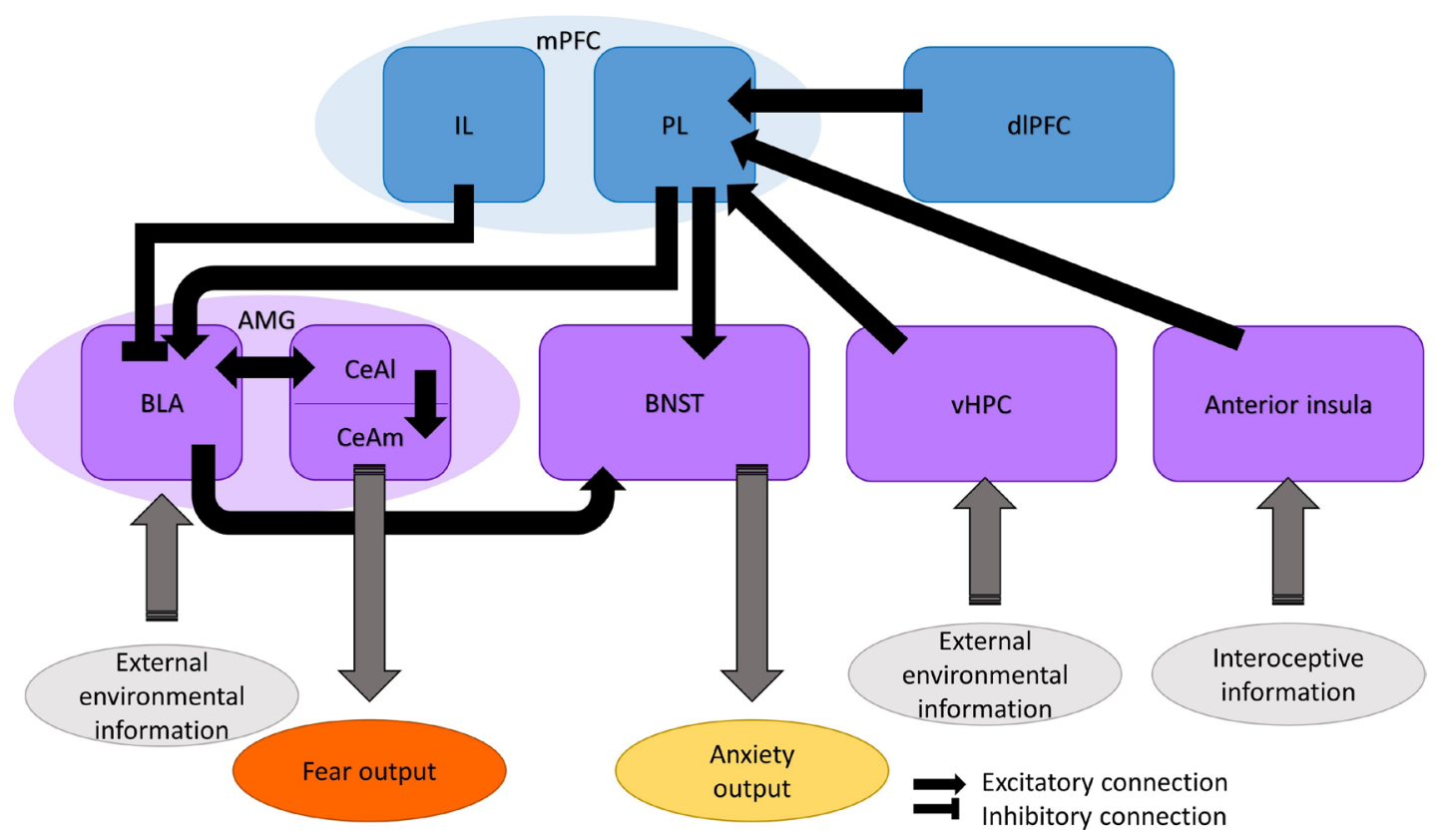

Figure 1 A simplified diagram of fear/anxiety circuitry, derived from animal research. A more detailed diagram of part of this circuitry can be found in Calhoon and Tye, 2015. Note that the dIPFC is included on the basis of research in non-human primates: rodents may not have a region homologous with the dIPFC. AMG, amygdala; BLA, basolateral amygdala; BNST, bed nucleus of the stria terminalis; dIPFC, dorsolateralprefrontal cortex; IL, infralimbic cortex; mPFC, medialprefrontal cortex; PL, paralimbic cortex; vHPC, ventral hippocampus; CeAl, lateral part of the central nucleus of the amygdala; CeAm, medial part of the central nucleus of the amygdala. 


\begin{tabular}{|c|c|}
\hline Paradigm & Description \\
\hline Threat of shock & $\begin{array}{l}\text { Threat-of-shock paradigms instruct participants who may be in one of two conditions: 'safe from shock' (during which no shocks are received) or } \\
\text { 'at risk of shock' (during which shocks may be received). Shocks may occur during the 'at-risk' blocks but with a low probability and generally are } \\
\text { scheduled independently of task performance. Other tasks may be performed with blocks of these contexts to investigate the effects of this sustained } \\
\text { threat on other cognitive processes. This is often measured using the eyeblink reflex, in a procedure known as 'startle'. Greater anxiety is thought to } \\
\text { correspond to the augmentation of the eyeblink reflex. }\end{array}$ \\
\hline Low dose $(7.5 \%)$ of $\mathrm{CO}_{2}$ & $\begin{array}{l}\text { Inhaling air with }>7.5 \% \mathrm{CO}_{2} \text { concentration over a period of around } 20 \text { minutes increases both subjective experience of and physiological symptoms } \\
\text { associated with anxiety. }\end{array}$ \\
\hline Darkness & $\begin{array}{l}\text { As humans are a diurnal species, this task elicits anxiety and potentiates anxious responses (eg, startle) by contrasting lighted conditions with } \\
\text { complete darkness. }\end{array}$ \\
\hline Vigilant threat monitoring & $\begin{array}{l}\text { Participants observe a line fluctuating on the screen which they are told reflects their own physiological levels of 'anxiety' (though in fact this } \\
\text { line is generated by experimenters), and when this line passes a threshold they will receive a shock. They are instructed to remain calm and avoid } \\
\text { accumulating shocks. }\end{array}$ \\
\hline Context conditioning & $\begin{array}{l}\text { During normal conditioning procedures, conditioning occurs either to the stimulus (cued conditioning) and/or to the context (context conditioning). } \\
\text { The context here refers to features of the environment that are present during the conditioning procedure. Context conditioning is separate to the } \\
\text { typical 'cued conditioning' used in fear learning paradigms due to the decreased temporal precision of the association between the context and the } \\
\text { unconditioned stimulus. }\end{array}$ \\
\hline Long-duration CS & $\begin{array}{l}\text { This paradigm is similar to typical fear conditioning, in that a predictive stimulus (CS) is paired with an unconditioned aversive stimulus (US), except } \\
\text { that the duration of presentation of the CS is longer, typically upwards of } 30 \text { seconds. }\end{array}$ \\
\hline $\begin{array}{l}\text { Approach-avoidance } \\
\text { conflict }\end{array}$ & $\begin{array}{l}\text { This type of paradigm, a translation of the animal paradigm of operant conflict, involves a choice or conflict between approaching rewards and } \\
\text { avoiding punishments, and has been argued to be anxiogenic. }\end{array}$ \\
\hline
\end{tabular}

These paradigms often include aversive stimuli such as shocks, but may also be used with other aversive stimuli such as aversive noises/pictures or blasts of cold air. The commonality among all these paradigms is that they all evoke a sustained anxiety state.

CS, conditioned stimuli.

with disorders that feature anxiety. ${ }^{7,512}$ Specifically, multiple studies have now shown exaggerated anxiety-potentiated (but not fear-potentiated) startle during unpredictable threat in $\mathrm{PD}^{\mathrm{s} 13-}$ ${ }^{s 16}$ and in post-traumatic stress disorder (PTSD). ${ }^{\text {s17-s19 }}$ GAD does not follow quite the same pattern-it is associated with increased startle overall during a threatening experimental environment. Exaggerated anxiety-potentiated startle may, therefore, constitute a risk factor or early biomarker for developing disorders including heightened anxiety. ${ }^{\text {s18,s19 }}$

Findings from unpredictable threat of shock studies in humans have implicated many of the same behavioural and neural responses that underpin (adaptive) anxiety in animals. In turn, these responses have also been implicated in pathological anxiety in humans. For instance, early functional MRI (fMRI) studies demonstrated amygdala involvement in processing both predictable $^{\mathrm{s} 20}$ and unpredictable threat. ${ }^{9}$ Activity in the amygdala was also shown to be elevated in those with social phobia relative to controls, ${ }^{\mathrm{s} 21,522}$ and it was argued that this elevated response was critical for the development of the excessive anxiety.

Subsequent work has, however, tempered these conclusions by demonstrating that the amygdala is sensitive to appetitive as well as aversive stimuli. ${ }^{\mathrm{s} 23}$ Moreover, consistent with animal data, many studies fail to report selective amygdala activation during sustained anxiety symptoms. ${ }^{.24,525}$ It has therefore been argued that perhaps a key role of the amygdala is in goal-directed cognitive processing and/or behaviour toward relevant/salient information. ${ }^{526}$ Given the clear asymmetries between appetitive and aversive stimuli value (i.e. the consequences are generally worse if one misses a threat than a reward), it may be that prior work associating the amygdala with anxiety reflects a more fundamental role of the amygdala that happens to be correlated with symptoms of anxiety (e.g. elevated harm avoidance in anxiety promoting the relevance/salience of threats) rather than any selective role in anxiety.

Animal models also highlight the role of the PFC in anxiety. In humans, elevated fMRI activity in dorsal regions of the PFC (dorsomedial PFC (dmPFC) and dorsal anterior cingulate (dACC)) has been associated with both unpredictable threat processing and pathological anxiety. ${ }^{.27}$ It has been argued, moreover, that it is specifically the rostral part of the dmPFC that drives conscious threat appraisal and worry. ${ }^{\mathrm{s} 28}$ In healthy humans, dACC/dmPFC activation has been associated with a reduced ability to extinguish fear responding, ${ }^{\text {s29 }}$ and patients with PTSD show increased activation in the dACC. ${ }^{.30}$ As with the amygdala, again, it should be noted that the $\mathrm{dACC} / \mathrm{dmPFC}$ is responsible for a wide range of functions including goal-directed behaviour, vestibular function, social responding and interoception. ${ }^{331}$ Critically, it is hyperactive in most psychiatric disorders ${ }^{\mathrm{s} 32}$ and, as with the amygdala, this may be due to a more generic role in anticipating emotional stimuli ${ }^{\mathrm{s33}}$ and hence a key role in appraising and expressing behavioural responses to the level of environmental threat. ${ }^{534}$ In other words, hyperactivity in psychiatric disorders probably reflects this region's more fundamental role-such as directing cognitive processing and/or behaviour toward relevant/salient information ${ }^{\mathrm{s} 2}$ - that is important for harm avoidance.

Perhaps unsurprisingly given the links between both amygdala and dACC/dmPFC activity and anxiety-relevant symptoms, connectivity between these regions has also been implicated in the pathophysiology of anxiety. Specifically, functional imaging studies show that connectivity between the amygdala and the mPFC increases when healthy individuals are exposed to threat of unpredictable shock, and that the strength of this connectivity is greater in individuals with higher dispositional anxiety. ${ }^{355-337}$ This extends to the pathological state, such that in those with social anxiety or GAD, this same circuitry displays greater connectivity without overt anxiety induction. ${ }^{.38}$ As such, the same circuitry that drives heightened attention toward threats under adaptive anxiety is also critical in pathological anxiety. To this end, it has been argued that this circuitry represents a human (functional) homologue of the rodent PL-amygdala/BNST circuit highlighted above. ${ }^{.30,534-537}$ If so, the key role that such circuitry plays in behavioural response to salient aversive information could underlie the harm avoidant negative bias toward threats in pathological anxiety. 
As also highlighted in the animal work above, the hippocampus is involved in anxiety, due, perhaps, to its key role in contextual learning/memory ${ }^{\mathrm{s} 39}$ and prospection ${ }^{\mathrm{s} 40}$ or, alternatively, a role in avoidance. ${ }^{24,541,542}$ Consistent with functional differentiation along the longitudinal axis of the rodent hippocampus, ${ }^{\text {s43 }}$ theta activity $(2-8 \mathrm{~Hz})$ from anterior hippocampus (or ventral in rodents) correlates with anxiety level in humans ${ }^{58}$; and at the same time theta from posterior hippocampus correlates with spatial memory performance in a simulated human version of the rodent Morris water maze task. ${ }^{.44}$ Notably, this thetabased coupling between hippocampus and MPFC scales up with increased threat probability ${ }^{45}$ and patients with PTSD exhibit aberrant activity and connectivity involving the hippocampus in the resting state. ${ }^{546}$ This theta coupling evidence comes from magnetoencephalography (MEG): the evidence is more mixed in fMRI, with both hypoactivation and hyperactivation of the hippocampus reported in patients. ${ }^{547}$ Finally, threat of shock also modulates memory performance, improving contextual learning under threatening conditions, ${ }^{s 4,549}$ which may be one mechanism by which the hippocampus maintains traumatic memories in disorders which feature elevated anxiety such as PTSD. ${ }^{550}$ Avoidance, on the other hand, can be studied using approach-avoidance conflict tasks (a variant on operant conflict tasks outlined above). Such tasks have been translated from non-human primate work ${ }^{551,552}$ into humans. ${ }^{24,541,542,553}$ This paradigm incorporates a range of tasks that set up a conflict between the inherent bias to avoid (learnt or prepotent) negative outcomes and an approach response. This is thought to either be anxiogenic in itself, ${ }^{24,541,442}$ or, at the very least, to elicit avoidance responses ${ }^{\mathrm{s} 54}$ which are a core feature of anxiety disorders. Critically, such tasks implicate the hippocampus ${ }^{24,541,542}$ and this conflict has been shown to be exacerbated, leading to increased avoidance, in humans during induced anxiety, ${ }^{\mathrm{s} 55}$ and in pathological anxiety. ${ }^{556}$

The insula also plays a complex role in anxiety in humans. Together with the ACC and mPFC, the insula is a part of a network that detects, interprets and reacts to internal bodily signals. ${ }^{557}$ Interoceptive signals are thought to be integrated in the insula following a posterior-mid-anterior pattern, with processing in the anterior insula producing conscious awareness of the information. ${ }^{558}$ The anterior insula has been suggested to make a key contribution to the anticipation and emotional experience of aversive stimuli, and, via the ACC, to the allocation of attention and initiation of appropriate action. ${ }^{{ }^{557}}$ Experimental psychopathology studies show that the anterior insula is broadly involved in anticipation of aversive events, but more specifically during anticipation of unpredictable compared with predictable threat $^{559}$ and sustained versus transient anticipation. ${ }^{9}$ Thus, the anterior insula is one of the structures that reliably maintains sustained activation during experimentally induced anxiety. ${ }^{9}$ It is also hyperactive in pathological anxiety, including $\mathrm{PD}^{\mathrm{s} 60}$ and $\mathrm{GAD},{ }^{\mathrm{s} 61}$ during sustained threat. In this instance, pathophysiology is not associated with chronic activation of the anterior insula but rather with heightened response during anxiety induction, possibly reflecting a feeling of lack of control ${ }^{\mathrm{s} 62}$ as well as autonomic and emotional distress during threat. ${ }^{\mathrm{s} 23}$

While animal studies have implicated the BNST in anxiety for some time now, it is only recently that progress in the spatial resolution of MRI has led to comprehensive exploration of this small structure in humans. ${ }^{563,564}$ Older studies found activity in regions overlapping the BNST during induced anxiety-that is, unpredictable threats ${ }^{9,59,524}$-as distinct from amygdala activity during predictable threats (ie, fear)..$^{9,65}$ In addition, BNST activation during unpredictable shock correlates positively with the magnitude of autonomic arousal. ${ }^{\mathrm{s} 62}$ Consistent with findings from startle studies, heightened sensitivity to unpredictable threat in PTSD and PDs is associated with elevated sustained BNST activation, making this structure a promising biomarker of disease and treatment targeting. ${ }^{s 60,566}$ These studies also identified several structures that are coactivated with the BNST, including the dmPFC, ventrolateral PFC, dIPFC and anterior insula, attesting to the complexity of a putative 'anxiety network'. These results suggest an important role for the BNST in mediating the hyperarousal and hypervigilance symptoms of pathological anxiety.

Additionally, high-resolution imaging using resting-state fMRI has revealed connectivity of the BNST in humans to many of the other regions discussed above. ${ }^{\mathrm{s} 64,667}$ Critically, anxiety induced by threat of shock reveals reduced BNST connectivity with the ventromedial prefrontal cortex and ACC. ${ }^{s 63}$ These are early findings, and research exploring clinical anxiety with high-resolution imaging is still in its infancy, but given the highlighted role of the BNST in the animal literature, clinical research targeting the BNST, its microcircuits and its functional connectivity offers great promise in the development of novel therapeutic interventions to treat pathological anxiety. ${ }^{32}$

Finally, as in non-human primates, the dIPFC is involved in anxiety regulation and dispositional anxiety in humans, ${ }^{\mathrm{s} 68}$ potentially because of a role in emotion regulation and attention control. In healthy subjects, the dlPFC is activated during anxiety induction procedures and the strength of this activation is negatively correlated with anxiety, indicating that poor dIPFC activation is associated with less anxiety downregulation (although this is speculative) ${ }^{569}$ Moreover, the dIPFC plays a role in explicit emotion regulation (for a review across all types of regulation strategies, see ${ }^{570}$ ). When subjects perform a cognitive task that engages the dlPFC (e.g. a WM task—a 'distraction' form of emotional regulation), dlPFC activation concomitantly reduces anxiety induced by unpredictable shock, via top-down control exerted on the dACC and ventrolateral PFC. ${ }^{\mathrm{s} 71}$ The dIPFC is, therefore, a key structure for healthy functioning. When the dIPFC is activated to keep task goals in mind, dIPFC engagement also suppresses emotional interference and alleviates anxiety. It is now well-established that the dIPFC is hypoactivated in those with psychiatric disorders featuring anxiety during cognitive tasks, emotion regulation studies and anxiety induction procedures..$^{.32,572}$

To summarise, research shows overlapping neural circuitry in response to anxiety between humans and animals. Specifically, connectivity between the hippocampus, BNST amygdala and medial prefrontal/cingulate cortex may contribute to a putative 'anxiety network' that may also be (down)regulated by dlPFC. Notably, this network of regions bears some similarity to the 'salience network', which incorporates regions such as the dACC and insular regions. ${ }^{573}$ Nevertheless, many of the regions (especially the PFC) are not clear translational homologues across species and, perhaps more importantly, human work highlights that many of these patterns of activity are not unique to anxiety and may instead reflect more fundamental cognitive processes, such as salience processing (in accordance with the key identity of many of those nodes within the salience network), which happen to constitute a key facet of anxiety.

\section{ROLE OF TREATMENT ON ANXIETY CIRCUITRY}

Characterising the behavioural effects of current treatments for pathological anxiety as well as their underlying neural 
mechanisms is crucial for the development of new treatments and the improvement of existing ones.

Cornwell et al (2017) recently reported MEG evidence that hypervigilant responding under threat of unpredictable shocks ${ }^{\text {s74 }}$ is reduced by the benzodiazepine alprazolam ${ }^{\mathrm{s} 75}$ and that this is driven by increased feedback signalling from ventrolateral prefrontal to sensory cortices. Although not directly implicating a role for any of the structures reviewed above, these data point to possible alternative avenues to examine the efficacy of novel anxiolytic treatments.

When given chronically to healthy individuals, the selective serotonin reuptake inhibitor (SSRI) citalopram selectively reduces anxiety-potentiated startle (but not fear-potentiated startle) to unpredictable threat. ${ }^{576}$ Interestingly, acute citalopram increases anxiety-potentiated startle, ${ }^{\mathrm{s} 77}$ an effect consistent with the clinical observations of transiently increased anxiety during initial SSRI treatment. ${ }^{\text {s7 }}$ Both the anxiolytic effects of chronic SSRI administration and the anxiogenic effects of acute SSRI administration have been replicated in rodents ${ }^{\text {s9 }}$ and the current view is that these effects involve the $\mathrm{BNST}^{7}$ and result from interactions between serotonin and corticotropin-releasing factors (CRFs). ${ }^{7, s 79}$

Acute tryptophan depletion (ATD) is a dietary manipulation that can be used to temporarily reduce levels of serotonin. ATD is associated with increased engagement of the dmPFC-amygala circuit that has been shown to be hyperengaged by both threat of shock $\mathrm{s}^{\mathrm{s} 35}$ and pathological anxiety ${ }^{\mathrm{s} 38}$ in humans. SSRIs may therefore work, at least in part, by elevating synaptic serotonin availability and hence reducing engagement of this dorsal prefrontal-amygdala circuit. ${ }^{\mathrm{s} 0}$ Indeed, it has been argued that the magnitude of SSRI response is predicted by greater pretreatment reactivity to threats in pregenual ACC and lesser reactivity in the amygdala. ${ }^{81}$ In anxious patients, SSRIs have also been shown to attenuate connectivity between the BNST and limbic and paralimbic structures. ${ }^{\text {s }}$ One hypothesis, therefore, is that SSRIs work by attenuating putative 'anxiety network' hyperactivity, ${ }^{\text {s83 }}$ but this remains to be tested.

Another broad class of treatment for anxiety disorders is psychological interventions. The goal of the most common psychological intervention, cognitive-behavioural therapy (CBT), is to attenuate negative mood states through cognitive reappraisal and emotion regulation strategies. ${ }^{\text {s4 }}$ Medial prefrontal and amygdala activity have been argued to predict treatment response to CBT in anxiety, ${ }^{\mathrm{s} 5}$ perhaps through modulation of circuitry between the mPFC and amygdala. Indeed, a recent meta-analysis suggested that the most robust predictors in response to therapy were significant post-therapy decreases in anterior cingulate/paracingulate gyrus, inferior frontal gyrus and insula activity. ${ }^{\text {s86 }}$

One problem with cross-sectional observational studies, however, is that it is unclear if neural circuitry change is specifically due to the intervention or rather reflects a generic change in symptoms (ie, it reflects symptom change, not mechanistic change). One way to study this is to explicitly modulate basic processes targeted by CBT in the absence of symptom changes. To this end, in healthy individuals, simple attentional instruction can alter the engagement of threat of shock-induced dmPFCamygdala circuitry. ${ }^{\text {s37 }}$ Specifically, asking individuals to re-appraise emotional stimuli as neutral dampens down activity in the circuitry thought to drive heightened response to threats in anxiety. Ongoing work is exploring whether this is a mechanism by which CBT for anxiety works.

Exposure is another important psychological intervention, with similar efficacy to $\mathrm{CBT}^{\mathrm{s}}{ }^{87}$ Exposure is primarily targeted at fear responding (eg, phobias), but can also be used to reduce anxiety. A more detailed review on findings from extinction paradigms-extinction is thought to be the mechanism by which exposure therapy works-in both humans and animals can be found in Milad and Quirk, ${ }^{\text {s88 }}$ but studies have implicated some of the same circuitry-activity was reduced in the amygdala, insula and anterior cingulate, but increased in the $\mathrm{dlPFC}^{89}$ following extinction.

In sum, preliminary work suggests that current treatments for anxiety may be effective through modulation of the translational circuitry outlined above. This work is in its infancy, but ultimately, a mechanistic understanding of treatment response may eventually enable improvement of existing treatments, better targeting of existing treatments to patients who will respond and provide targets for the design of novel treatments, hence resulting in increased recovery rates for anxiety disorders.

\section{FUTURE PERSPECTIVES}

1. One key problem with the current categorical disease classifications for anxiety disorders is that of heterogeneity within diagnostic categories and overlapping symptoms across disorders. Specifically, anxiety seems to be an obvious candidate for an RDoC-based approach, with the RDoC matrix highlighting several constructs (acute threat, potential threat (anxiety) and sustained threat) that map onto the distinction made in this article between anxiety and fear. This distinction may map onto different types of vulnerability: perhaps one associated with predictable threat (ie, fear) and the other with unpredictable threat (ie, anxiety). Of relevance, this distinction appears in line with data of factor analytic studies showing a distinction for 'fear disorder' and 'anxiousmisery' in the anxiety disorders. ${ }^{.90}$ Large-scale studies will be necessary to obtain sufficient power to determine the extent to which anxiety circuitry is compromised (in similar and in different ways) across psychiatric and neurological disorders, which could improve classification, treatment planning and pave the way for precision medicine approaches. Anxious patients with heightened sensitivity to unpredictable threat (e.g. $\mathrm{PD}^{\mathrm{s} 13}$ ), for example, may particularly benefit from treatment that downregulate the anxiety circuit (eg, SSRIs ${ }^{576}$ ). This approach can be applied more broadly: those with elevated sensitivity to unpredictable threat, whatever their psychiatric/neurological diagnosis, may benefit from the same treatments.

2. The BNST has long been overlooked. It is a small but functionally complex structure comprised of at least 18 subregions $^{36}$ involved, among other functions, in opposing anxiolytic and anxiogenic circuits. Basic research in rodents on intrinsic circuits of the BNST and how these circuits are impacted by stress hormones and neurotransmitters will be crucial to increase our knowledge of normal and pathological anxiety and to develop treatment. ${ }^{\mathrm{s} 91}$ For example, the actions of neuropeptide CRF on the BNST in relation to sustained anxiety provided a strong rationale for the therapeutic development of CRF1 antagonists to treat mood and anxiety disorders. However, these have failed in human models ${ }^{\mathrm{s} 92}$ and in clinical trials. ${ }^{593}$ More studies on the local and more distal connectivity of the BNST will be necessary to understand why CRF antagonists are anxiolytic in animal models but not in humans. On this note, a question remains about how best to translate our understanding of such subunits into humans. Improved, higher resolution, fMRI techniques will undoubtedly help, but fMRI signal is still several steps 
away from the underlying neural activity. Work with specific positron-emission tomography ligands and even direct electrical recording from those with clinical implants may be needed to ultimately corroborate translational patterns.

3. Relatedly, direct translation across humans and animals is difficult. Animal models are essential to advance mechanistic understanding of behaviours, but their limitations must be acknowledged. Pathological anxiety is, above all, a disorder of feelings supported by conscious and unconscious experiences, and animal models rely on overt behaviour and physiological measures, rather than cognitions or subjective experiences. Additionally, many processes, for example, the psychological symptoms targeted by CBT cannot be directly studied in animal models, and many regions-especially cortical areas-are likely not direct functional homologues across animal models (if they exist at all in animals with smaller cortices). This is of particular concern because many animal models of treatment have failed to translate to humans. To overcome this, future work may seek to use models of anxiety in healthy humans alongside the exact same models in animals as putative anxiolytic drug screens to ensure successful translation. Furthermore, cross-fertilisation may be important where roadblocks in treatment development occur: theories and biological insights from one type of research may produce advances in the other.

4. Beyond pharmacology, neurostimulation research is starting to bear exciting results. Deep brain stimulation of the BNST reduces anxiety in a rodent model and in humans. ${ }^{594}$ Repetitive transcranial magnetic stimulation (rTMS) is a less invasive neurostimulation technique. As low-frequency oscillations within cortical networks decrease cortical excitability, ${ }^{992}$ targeting the dmPFC or dlPFC-amygdala coupling with low $(1 \mathrm{~Hz})$ rTMS could downregulate activity in this circuit and ultimately reduce negative affect. rTMS of the dIPFC is approved by the Food and Drug Administration as a secondline treatment for depression, and research is currently undergoing to examine its effectiveness in anxiety. ${ }^{\mathrm{s} 95}$

5. Future research should also focus on the emergence of pathological anxiety, ideally taking a developmental perspective. There are a number of difficulties inherent in developmental work in anxiety, with the most important being the ethical issues with exposing children and adolescents to aversive events such as shock. ${ }^{996}$ However, researchers have recently successfully collected data on fear-potentiated startle in adolescents using alternative aversive stimuli such as cold air blasts or aversive screams. ${ }^{\text {s96,997 }}$ These are promising steps toward creating paradigms that can elucidate the developmental basis of anxiety.

\section{CONCLUDING REMARKS}

Our understanding of anxiety circuitry has grown considerably thanks to experimental psychopathology models exploring the impact of unpredictable threats. Consistent with animal models, fear and anxiety in humans may be mediated by dissociable, although partly overlapping, neural mechanisms. Structures implicated in anxiety but not fear include the BNST, hippocampus, dmPFC, insula and dlPFC. Fear-related and anxietyrelated defensive responses are mediated by the CeA and BNST, respectively.

From a clinical and treatment perspective, there is overlap in the circuitry implicated in disorders featuring anxiety and in the mechanism of action of reference anxiolytics. Pathophysiological mechanisms are found in the same neural structures that respond adaptively to anxiety induction procedures in healthy humans. Neural dysfunction can take two forms, chronic activation (inappropriate activation in the absence of anxiety induction challenge, for example, heightened amygdala-dmPFC connectivity) or exaggerated activation in response to an unpredictable threat (i.e. insula).

Despite this progress, much remains to be learnt. With advances in technologies in basic (optogenetic, molecular biology, transgenic and knockout mice) and clinical research (high spatial resolution of fMRI, better statistical tools), the focus should be on improving knowledge of local microcircuits and neural oscillations among distant regions that supports behaviour. Clinically, research on fear and anxiety circuitry might be used to create an evidence-based nosology. ${ }^{\text {s18 }}$ Ultimately, improved, personalised and new treatment strategies will be difficult to develop without a better understanding of the underlying mechanisms of anxiety; the work reviewed here constitutes a step forward, but a precise mechanistic understanding is still far off.

Additional references can be found in supplementary file.

Contributors OJR, ACP, BC and CG are all responsible for drafting and editing this manuscript.

Funding This work was supported by the personal fellowships MR/K024280/1 and MR/R020817/1 from the Medical Research Council to OJR and the Intramural Research Program of the National Institute of Mental Health, project number ZIAMH002798 (clinical protocol 02-M-0321 (NCT00047853), 01-M-0185 (NCT00026559)) to CG. OJR has completed consultancy work for IESO digital health and Brainbow and is running an Investigator Initiated Trial with Lundbeck. He holds an MRC Industrial Collaboration Award with Cambridge Cognition.

Competing interests OJR has completed consultancy work for leso Digital Health and Brainbow and is running an Investigator Initiated Trial with Lundbeck. He holds an MRC Industrial Collaboration Award with Cambridge Cognition.

Patient consent for publication Not required.

Provenance and peer review Commissioned; internally peer reviewed.

\section{ORCID iDs}

Oliver J Robinson http://orcid.org/0000-0002-3100-1132

Alexandra C Pike http://orcid.org/0000-0003-1972-5530

\section{REFERENCES}

1 Greenberg PE, Kessler RC, Birnbaum HG, et al. The economic burden of depression in the United States: how did it change between 1990 and 2000? J Clin Psychiatry 2003;64:1465-75.

2 Baldwin DS, Huusom AKT, Maehlum E. Escitalopram and paroxetine in the treatment of generalised anxiety disorder: randomised, placebo-controlled, double-blind study. Br J Psychiatry 2006;189:264-72.

3 Kim JJ, Jung MW. Neural circuits and mechanisms involved in Pavlovian fear conditioning: a critical review. Neurosci Biobehav Rev 2006;30:188-202.

4 Tovote P, Fadok JP, Lüthi A. Neuronal circuits for fear and anxiety. Nat Rev Neurosci 2015;16:317-31

5 Fullana MA, Harrison BJ, Soriano-Mas C, et al. Neural signatures of human fear conditioning: an updated and extended meta-analysis of fMRI studies. Mol Psychiatry 2016;21:500-8.

6 Davis M. Are different parts of the extended amygdala involved in fear versus anxiety? Biol Psychiatry 1998;44:1239-47.

7 Davis M, Walker DL, Miles L, et al. Phasic vs Sustained Fear in Rats and Humans: Role of the Extended Amygdala in Fear vs Anxiety. Neuropsychopharmacol 2010;35:105-35

8 Barlow DH. Unraveling the mysteries of anxiety and its disorders from the perspective of emotion theory. Am Psychol 2000;55:1247-63.

9 Alvarez RP, Chen G, Bodurka J, et al. Phasic and sustained fear in humans elicits distinct patterns of brain activity. Neuroimage 2011:55:389-400.

10 Lázaro-Muñoz G, LeDoux JE, Cain CK. Sidman instrumental avoidance initially depends on lateral and basal amygdala and is constrained by central amygdalamediated Pavlovian processes. Biol Psychiatry 2010;67:1120-7.

11 Evans DA, Stempel AV, Vale R, et al. A synaptic threshold mechanism for computing escape decisions. Nature 2018;558:590-4.

12 Insel T, Cuthbert B, Garvey M, et al. Research domain criteria (RDoC): toward a new classification framework for research on mental disorders. Am J Psychiatry 2010;167:748-51. 
13 NIMH Research Domain Criteria (RDoC). project Negative Valence Systems: Workshop Proceedings, 2011.

14 American Psychiatric Association. Diagnostic and statistical manual of mental disorders (DSM-5). 5th ed. Arlington, VA, US: American Psychiatric Publishing, 2013.

15 Phelps EA, LeDoux JE. Contributions of the amygdala to emotion processing: from animal models to human behavior. Neuron 2005;48:175-87.

16 Grillon C, Baas JMP, Pine DS, et al. The benzodiazepine alprazolam dissociates contextual fear from cued fear in humans as assessed by fear-potentiated startle. Biol Psychiatry 2006;60:760-6.

17 Baas JMP, Grillon C, Böcker KBE, et al. Benzodiazepines have no effect on fearpotentiated startle in humans. Psychopharmacology 2002;161:233-47.

18 Moberg CA, Curtin JJ. Alcohol selectively reduces anxiety but not fear: startle response during unpredictable versus predictable threat. J Abnorm Psychol 2009;118:335-47

19 Graham SJ, Scaife JC, Langley RW, et al. Effects of lorazepam on fear-potentiated startle responses in man. J Psychopharmacol 2005;19:249-58.

20 Blanchard RJ, Yudko EB, Rodgers RJ, et al. Defense system psychopharmacology: an ethological approach to the pharmacology of fear and anxiety. Behav Brain Res 1993;58:155-65.

21 Maier SF, Watkins LR. Stressor controllability, anxiety, and serotonin. Cognit Ther Res 1998:22:595-613.

22 Walz N, Mühlberger A, Pauli P. A human open field test reveals thigmotaxis related to agoraphobic fear. Biol Psychiatry 2016;80:390-7

23 Biedermann SV, Biedermann DG, Wenzlaff F, et al. An elevated plus-maze in mixed reality for studying human anxiety-related behavior. BMC Biol 2017;15.

24 Bach DR, Guitart-Masip M, Packard PA, et al. Human hippocampus arbitrates approach-avoidance conflict. Curr Biol 2014;24:541-7.

25 Porter VR, Buxton WG, Fairbanks LA, et al. Frequency and characteristics of anxiety among patients with Alzheimer's disease and related dementias. J Neuropsychiatry Clin Neurosci 2003;15:180-6.

26 Broen MPG, Narayen NE, Kuijf ML, et al. Prevalence of anxiety in Parkinson's disease: A systematic review and meta-analysis. Mov Disord 2016;31:1125-33.

27 Scholten AC, Haagsma JA, Cnossen MC, et al. Prevalence of and Risk Factors for Anxiety and Depressive Disorders after Traumatic Brain Injury: A Systematic Review. $J$ Neurotrauma 2016:33:1969-94.

28 Hart T, Fann JR, Chervoneva I, et al. Prevalence, Risk Factors, and Correlates of Anxiety at 1 Year After Moderate to Severe Traumatic Brain Injury. Arch Phys Med Rehabil 2016;97:701-7.

29 LeDoux JE. Emotion circuits in the brain. Annu Rev Neurosci 2000;23:155-84.

30 Treit D, Pesold C, Rotzinger S. Dissociating the anti-fear effects of septal and amygdaloid lesions using two pharmacologically validated models of rat anxiety. Behav Neurosci 1993;107:770-85.

31 Blanchard DC, Griebel G, Blanchard RJ. Mouse defensive behaviors: pharmacological and behavioral assays for anxiety and panic. Neurosci Biobehav Rev 2001;25:205-18.

32 Lebow MA, Chen A. Overshadowed by the amygdala: the bed nucleus of the stria terminalis emerges as key to psychiatric disorders. Mol Psychiatry 2016:21:450-63.

33 Heimer L, Harlan RE, Alheid GF, et al. Substantia innominata: a notion which impedes clinical-anatomical correlations in neuropsychiatric disorders. Neuroscience 1997;76:957-1006

34 Goode TD, Maren S. Role of the bed nucleus of the stria terminalis in aversive learning and memory. Learn Mem 2017;24:480-91.

35 Hammack SE, Todd TP, Kocho-Schellenberg M, et al. Role of the bed nucleus of the stria terminalis in the acquisition of contextual fear at long or short context-shock intervals. Behav Neurosci 2015;129:673-8.

36 Bota M, Sporns O, Swanson LW. Neuroinformatics analysis of molecular expression patterns and neuron populations in gray matter regions: the rat BST as a rich exemplar. Brain Res 2012;1450:174-93.

37 Duvarci S, Bauer EP, Paré D. The bed nucleus of the stria terminalis mediates interindividual variations in anxiety and fear. J Neurosci 2009:29:10357-61.
38 Kim S-Y, Adhikari A, Lee SY, et al. Diverging neural pathways assemble a behavioural state from separable features in anxiety. Nature 2013;496:219-23.

39 Jennings JH, Sparta DR, Stamatakis AM, et al. Distinct extended amygdala circuits for divergent motivational states. Nature 2013:496:224-8.

40 Avery SN, Clauss JA, Blackford JU. The human BNST: functional role in anxiety and addiction. Neuropsychopharmacology 2016;41:126-41.

41 Choi DC, Furay AR, Evanson NK, et al. Bed nucleus of the stria terminalis subregions differentially regulate hypothalamic-pituitary-adrenal axis activity: implications for the integration of limbic inputs. J Neurosci 2007;27:2025-34.

42 Roman CW, Lezak KR, Kocho-Schellenberg M, et al. Excitotoxic lesions of the bed nucleus of the stria terminalis (BNST) attenuate the effects of repeated stress on weight gain: evidence for the recruitment of BNST activity by repeated, but not acute, stress. Behav Brain Res 2012:227:300-4.

43 Dong H-W, Swanson LW. Projections from bed nuclei of the stria terminalis, posterior division: Implications for cerebral hemisphere regulation of defensive and reproductive behaviors. J Comp Neurol 2004;471:396-433.

44 Fendt M, Siegl S, Steiniger-Brach B. Noradrenaline transmission within the ventral bed nucleus of the stria terminalis is critical for fear behavior induced by trimethylthiazoline, a component of fox odor. J Neurosci 2005:25:5998-6004.

45 Ciocchi S, Herry C, Grenier F, et al. Encoding of conditioned fear in central amygdala inhibitory circuits. Nature 2010;468:277-82.

46 Haubensak W, Kunwar PS, Cai H, et al. Genetic dissection of an amygdala microcircuit that gates conditioned fear. Nature 2010;468:270-6.

47 Whalen PJ, Bush G, McNally RJ, et al. The emotional counting Stroop paradigm: a functional magnetic resonance imaging probe of the anterior cingulate affective division. Biol Psychiatry 1998:44:1219-28.

48 McDonald AJ. Cortical pathways to the mammalian amygdala. Prog Neurobiol 1998:55:257-332

49 Sotres-Bayon F, Quirk GJ. Prefrontal control of fear: more than just extinction. Curr Opin Neurobiol 2010;20:231-5.

50 Bi L-L, Wang J, Luo Z-Y, et al. Enhanced excitability in the infralimbic cortex produces anxiety-like behaviors. Neuropharmacology 2013;72:148-56.

51 Baeg EH, Kim YB, Jang J, et al. Fast spiking and regular spiking neural correlates of fear conditioning in the medial prefrontal cortex of the rat. Cereb Cortex 2001;11:441-51.

52 Burgos-Robles A, Vidal-Gonzalez I, Quirk GJ. Sustained conditioned responses in prelimbic prefrontal neurons are correlated with fear expression and extinction failure. J Neurosci 2009;29:8474-82.

53 Gilmartin MR, McEchron MD. Single neurons in the medial prefrontal cortex of the rat exhibit tonic and phasic coding during trace fear conditioning. Behav Neurosci 2005;119:1496-510.

54 Adhikari A, Topiwala MA, Gordon JA. Synchronized activity between the ventral hippocampus and the medial prefrontal cortex during anxiety. Neuron 2010;65:257-69.

55 Swanson LW. A direct projection from Ammon's horn to prefrontal cortex in the rat. Brain Res 1981:217:150-4.

56 Gray CM, König P, Engel AK, et al. Oscillatory responses in cat visual cortex exhibit inter-columnar synchronization which reflects global stimulus properties. Nature 1989;338:334-7.

57 von Stein A, Sarnthein J. Different frequencies for different scales of cortica integration: from local gamma to long range alpha/theta synchronization. Int $J$ Psychophysiol 2000;38:301-13.

58 Cornwell BR, Arkin N, Overstreet C, et al. Distinct contributions of human hippocampal theta to spatial cognition and anxiety. Hippocampus 2012;22:1848-59.

59 Ito R, Lee ACH. The role of the hippocampus in approach-avoidance conflict decision-making: Evidence from rodent and human studies. Behav Brain Res 2016:313:345-57.

60 Vertes RP. Differential projections of the infralimbic and prelimbic cortex in the rat. Synapse 2004;51:32-58. 